\title{
SEOM Clinical Guideline for the treatment of pancreatic cancer (2016)
}

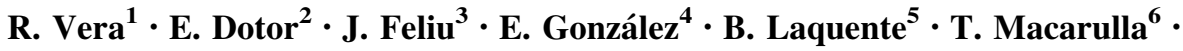 \\ E. Martínez ${ }^{7} \cdot$ J. Maurel $^{8} \cdot$ M. Salgado ${ }^{9} \cdot$ J. L. Manzano ${ }^{10}$
}

Received: 13 November 2016/Accepted: 15 November 2016/Published online: 28 November 2016

(C) The Author(s) 2016. This article is published with open access at Springerlink.com

\begin{abstract}
Pancreatic cancer remains an aggressive disease with a 5 year survival rate of $5 \%$. Only $15 \%$ of patients with pancreatic cancer are eligible for radical surgery. Evidence suggests a benefit on survival with adjuvant chemotherapy (gemcitabine o fluourouracil) after R1/R0 resection. Adjuvant chemoradiotherapy is also a valid option in patients with positive margins. Borderline resectable pancreatic cancer is defined as the involvement of the mesenteric vasculature with a limited extension. These tumors are technically resectable, but with a high risk of positive margins. Neoadjuvant treatment represents the best option for achieving an R0 resection. In advanced disease, two new chemotherapy treatment schemes (Folfirinox or Gemcitabine plus nab-paclitaxel) have showed improvements in
\end{abstract}

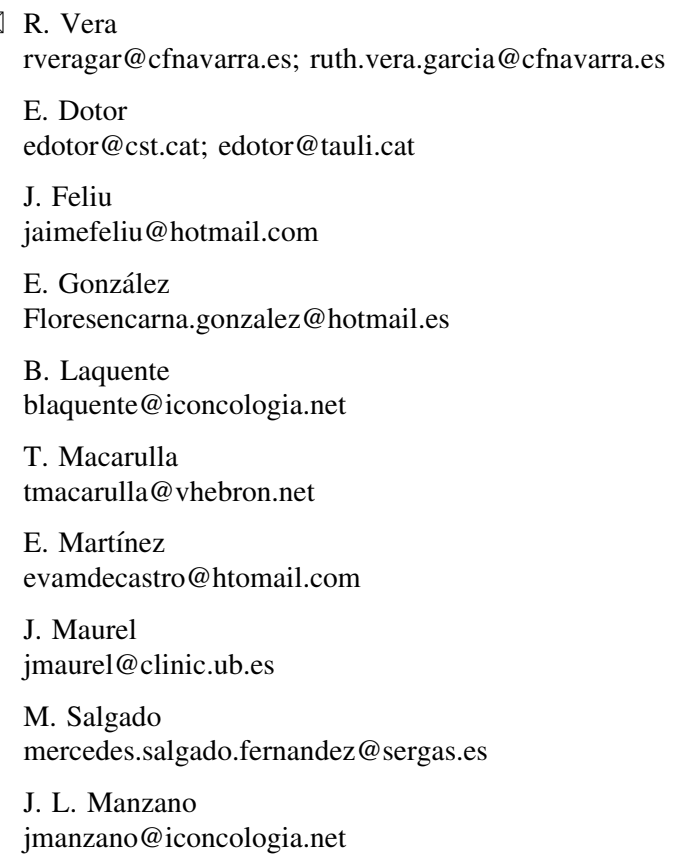
overall survival compared with gemcitabine alone. Progress in pancreatic cancer treatment will require a better knowledge of the molecular biology of this disease, focusing on personalized cancer therapies in the near future.

Keywords Pancreatic cancer - Treatment - Diagnostic . Guidelines

\section{Introduction}

Pancreatic cancer is a major health problem. Despite not having a high incidence in the population, its aggressive behavior leads to a high mortality [1]. In Spain, 6367 new

1 Department of Medical Oncology, Complejo Hospitalario de Navarra, c/Irunlarrea-3, 31008 Pamplona, Spain

2 Consorcio Sanitario de Terrassa, Barcelona, Spain

3 Hospital Universitario la Paz, Madrid, Spain

4 Complejo Hospitalario Universitario de Granada Virgen de las Nieves, Granada, Spain

5 ICO-Hospitalet de LLobregat, Hospital Duran i Reynals, Hospitalet de Llobregat, Spain

6 Hospital Vall'Hebron, Barcelona, Spain

7 Hospital Universitario Marqués de Valdecilla, Santander, Spain

8 Hospital Clínic i Provincial de Barcelona, Barcelona, Spain

9 Complexo Hospitalario de Orense (CHUO), Ourense, Spain

10 ICO-Badalona, Hospital Germans Trias i Pujol, Barcelona, Spain 
cases were diagnosed in 2012 (3\% of global cancer incidence), 3335 in men and 3032 in women. Pancreatic cancer was the fourth most fatal cancer (5720 cases) in Spain, after lung, colorectal, and breast cancer. In 2012, approximately 3000 men and 2700 women died from this disease [2]. Most patients who are diagnosed with pancreatic cancer are aged between 65 and 70 years old. It is rare that this tumor appears below 60 years of age; in this case, we should rule out the association with a genetic alteration. Only a small proportion of pancreatic cancers are related to a genetic alteration (5-10\%). Germline mutations in BRCA2, p16, ATM, STK11, PRSS1/PRSS2, SPINK1, PALB2, and DNA mismatch repair genes are associated with varying degrees of increased risk for pancreatic carcinoma. Mutation in BRCA2 is probably the most common inherited disorder in familial pancreatic cancer [3]. Among the remaining 90\%, the major risk factors are tobacco, $H$. pylori infection, and factors related to dietary habits [4].

At the time of diagnosis, less than $20 \%$ of patients are suitable for resection, given the advanced stage of the disease. After surgical resection, survival rates usually are between 10 and 20 months. Defining the treatment strategy for patients suffering from pancreatic carcinoma requires a specialized multidisciplinary team.

\section{Diagnosis and staging}

\section{Diagnosis}

After a suspicion of pancreatic cancer based on signs and symptoms (weight loss, jaundice, pain or depression, among others), pathologic diagnosis is mandatory in unresectable and borderline resectable cases in which a preoperative treatment is planned.

A pathological diagnosis of PC is usually made with fine-needle aspiration (FNA) by endoscopic ultrasound (EUS) guidance or computed tomography (CT). EUS-FNA is preferred in cases of resectable and borderline resectable disease. Cytologic specimens have limitation for biomarkers studies and do not include stroma. Core needle biopsies (CNB), that use a slightly larger and hollow needle to withdraw small cylinders of tissue, could be more useful in the near future.

Diagnosis of pancreatic cancer should include cytologic or pathologic diagnosis [5], staging (Table 1), and evaluation of the patient basal situation (PS, comorbidities...) and his preferences. Unnecessary delays should be avoided to treat the patient as soon as possible.
Table 1 Evaluations recommended for the proper staging of pancreatic cancer

Complete history and physical examination

Laboratory test: blood count and serum chemistry including PCR, albumin and levels of the carbohydrate antigen CA19-9

$\mathrm{CT}$ of the chest and abdomen

Histologic or cytologic diagnosis

Bone scan in presence of bone pain, elevated serum calcium or elevated alkaline phosphatase levels

In patients with resectable tumors (optional), border-line or locally advanced pancreatic cancer (mandatory)

EUS+FNA

In patients with borderline resectable tumors

Diagnostic laparoscopy will be assessed in cases of suspicion of peritoneal involvement (no consensus)

\section{Staging system}

The classification system most frequently used in pancreatic cancer is the tumor-node-metastasis (TNM) system of the combined American Joint Committee on Cancer (AJCC)/International Union Against Cancer (UICC). This staging system classifies tumors depending on the size and extent of the primary tumor $(\mathrm{T})$, the presence or absence of regional lymph node metastasis $(\mathrm{N})$ and the presence or absence of distant metastasis (M). The latest update is the 8th edition of this classification system published in 2016 and recently validated (Table 2) [6].

All patients with PC should be valued from the beginning at a multidisciplinary committee in a reference center with an adequate volume of patients, for decision-making regarding treatment, especially those with potential surgical indication. It is available a classification that allows to evaluate the potential resectability based on radiological findings (Table 3) [7, 8].

\section{Recommendations}

Laboratory test with CA19-9, CT chest and abdomen, histologic or cytologic diagnostic, EUS in resectable tumors (IV, C).

\section{Treatment}

\section{Resectable disease}

Surgery is the standard treatment for resectable disease (70\% of patients have positive margins independently of the quality of the surgical resection). 
Table 2 Staging group

\begin{tabular}{|c|c|c|c|}
\hline \multicolumn{4}{|c|}{ Primary tumor $(\mathrm{T})$} \\
\hline \multicolumn{4}{|c|}{$\mathrm{T} 1$ : Maximum tumor diameter $\leq 2 \mathrm{~cm}$} \\
\hline \multicolumn{4}{|c|}{ T2: Maximum tumor diameter $>2 \leq 4 \mathrm{~cm}$} \\
\hline \multicolumn{4}{|c|}{ T3: Maximum tumor diameter $>4 \mathrm{~cm}$} \\
\hline \multicolumn{4}{|c|}{$\begin{array}{l}\text { T4: Tumor involves the celiac axis or the superior mesenteric } \\
\text { artery (unresectable primary tumor) }\end{array}$} \\
\hline \multicolumn{4}{|c|}{ Regional lymph nodes (N) } \\
\hline \multicolumn{4}{|c|}{ N0: No regional lymph node metastasis } \\
\hline \multicolumn{4}{|c|}{ N1: Metastasis in 1-3 regional lymph nodes } \\
\hline \multicolumn{4}{|c|}{ N2: Metastasis in 4 regional lymph nodes } \\
\hline \multicolumn{4}{|c|}{ Distant metastases $(\mathrm{M})$} \\
\hline \multicolumn{4}{|c|}{ M0: No distant metastasis } \\
\hline \multicolumn{4}{|c|}{ M1: Distant metastasis } \\
\hline Stage & $\mathrm{T}$ & $\mathrm{N}$ & M \\
\hline 0 & Tis & No & M0 \\
\hline IA & $\mathrm{T} 1$ & No & M0 \\
\hline IB & $\mathrm{T} 2$ & No & M0 \\
\hline IIA & $\mathrm{T} 3$ & No & M0 \\
\hline IIB & $\mathrm{T} 1-3$ & N1 & M0 \\
\hline \multirow[t]{2}{*}{ III } & Any $\mathrm{T}$ & Any $\mathrm{N}$ & M0 \\
\hline & $\mathrm{T} 4$ & & \\
\hline IV & Any $\mathrm{T}$ & Any $\mathrm{N}$ & M1 \\
\hline
\end{tabular}

Patients with tumors located in the pancreatic head are treated with pancreatoduodenectomy (Whipple procedure). When the tumor is located in the body or tail of the gland, the surgical procedure is a distal pancreatectomy. In some cases, a total pancreatectomy may be required.

Even with a R0 resection, the recurrence rate is very high. Therefore, adjuvant treatment is required in almost all the patients with resected adenocarcinoma of the pancreas. It is advisable to start adjuvant therapy between 6 and 8 weeks after surgery.

Post-operative treatment in pancreatic cancer has been evaluated in several clinical trials.

CONKO-1 trial demonstrated that patients treated with adjuvant gemcitabine $\left(1000 \mathrm{mg} / \mathrm{m}^{2}\right.$ day $1,8,15 / 28$ days) for 6 months after surgery presented longer disease-free survival than those patients treated with surgery alone (13.4 vs. 6.9 months, $p<0.001$ ) [9]. The ESPAC-3 trial compared the administration of adjuvant chemotherapy with 6 months of either bolus fluorouracil $\left(425 \mathrm{mg} / \mathrm{m}^{2}\right.$ and folinic acid $20 \mathrm{mg} / \mathrm{m}^{2}$ day $1-5$ every 28 days) or gemcitabine. Median survival was equivalent in both arms (23 months, $p 0.39$ ), but gemcitabine was better tolerated [10].

As a result of these studies, gemcitabine and 5-fluorouracil can both be considered as the standard of adjuvant treatment in pancreatic cancer.

Table 3 Criteria defining resectability status according to NCCN Guidelines version 1.2016 (Pancreatic adenocarcinoma)

\begin{tabular}{|c|c|c|c|}
\hline $\begin{array}{l}\text { Resectability } \\
\text { status }\end{array}$ & Distant metastases & Arterial & Venous \\
\hline Resectable & No & $\begin{array}{l}\text { No arterial tumor contact [celiac axis (CA), superior } \\
\text { mesenteric artery (SMA) or common hepatic } \\
\text { artery (CHA)] }\end{array}$ & $\begin{array}{l}\text { No tumor contact with the superior mesenteric vein } \\
\text { (SMV) or portal vein (PV) or } \leq 180^{\circ} \text { contact } \\
\text { without vein contour irregularity }\end{array}$ \\
\hline $\begin{array}{l}\text { Boderline } \\
\text { resectable }\end{array}$ & No & $\begin{array}{l}\text { Head/uncinated process: } \\
\text { Solid tumor contact with CHA without extension to } \\
\text { CA or hepatic artery bifurcation allowing for safe } \\
\text { and complete resection and reconstruction } \\
\text { Solid tumor contact with the SMA of } \leq 180^{\circ} \\
\text { Body and tail: } \\
\text { Solid tumor contact with the CA of } \leq 180^{\circ} \\
\text { Solid tumor contact with the CA of }>180^{\circ} \text { without } \\
\text { involvement of the aorta and with intact and } \\
\text { uninvolved gastroduodenal artery }\end{array}$ & $\begin{array}{l}\text { Solid tumor contact with the SMV or PV of }>180^{\circ} \text {, } \\
\text { contact of } \leq 180^{\circ} \text { with contour irregularity of the } \\
\text { vein or thrombosis of the vein but with } \\
\text { suitable vessel proximal and distal to the site of } \\
\text { involvement allowing for safe and complete } \\
\text { resection and vein reconstruction } \\
\text { Solid tumor contact with the inferior vena cava } \\
\text { (IVC) }\end{array}$ \\
\hline Unresectable & $\begin{array}{l}\text { Yes (including } \\
\text { non-regional } \\
\text { lymph node } \\
\text { metastasis) }\end{array}$ & $\begin{array}{l}\text { Head/uncinated process: } \\
\text { Solid tumor contact with SMA }>180^{\circ} \\
\text { Solid tumor contact with } \mathrm{CA}>180^{\circ} \\
\text { Solid tumor contact with the first jejunal SMA } \\
\text { branch } \\
\text { Body and tail: } \\
\text { Solid tumor contact of }>180^{\circ} \text { with de SMA or CA } \\
\text { Solid tumor contact with the CA and aortic } \\
\text { involvement }\end{array}$ & $\begin{array}{l}\text { Head/uncinated process: } \\
\text { Unreconstructible SMV/PV due to tumor } \\
\text { involvement or occlusion (can be due tumor or } \\
\text { bland thrombus) } \\
\text { Contact with most proximal draining jejunal branch } \\
\text { into SMV } \\
\text { Body and tail: } \\
\text { Unreconstructible SMV/PV due to tumor } \\
\text { involvement or occlusion (can be due tumor or } \\
\text { bland thrombus) }\end{array}$ \\
\hline
\end{tabular}


This year in the last ASCO meeting, preliminary results of ESPAC-4 trial were presented. Adjuvant gemcitabine at a standard dose was compared with the combination of gemcitabine $\left(1000 \mathrm{mg} / \mathrm{m}^{2}\right.$ day $1,8,15 / 28$ days $)$ and capecitabine $\left(1660 \mathrm{mg} / \mathrm{m}^{2} /\right.$ day $\mathrm{d} 1-21 / 28$ days $)$ for 6 months [11]. Median survival for patients treated with gemcitabine and capecitabine was significantly better than gemcitabine monotherapy (28 vs. 25.5 months, HR 0.82 , $p$ 0.032). The role of chemoradiotherapy has not been clearly established as beneficial because of the controversial results in previous studies, although it is an option in some individual cases with $\mathrm{R} 1$ resection [12].

\section{Recommendations}

1. Adjuvant treatment is required in all resected patients if the patient is recovered from the surgery (I, A).

2. Six cycles of gemcitabine or 5-fluorouracil is considered a standard treatment (I, A).

\section{Borderline resectable disease}

Numerous non-randomized studies in patients with marginally resectable disease that have used different treatment regimens with neoadjuvant chemotherapy or chemoradiation have managed to increase the resectability rate from 33 to $64 \%$ in borderline tumors. There is limited evidence to recommend specific neoadjuvant regimens off-study, and practices vary with regard to the use of chemotherapy and chemoradiation [13, 14].

The benefit of neoadjuvant therapies in borderline resectable pancreatic cancer has been retrospectively reviewed by different authors, with treatment regimens including 2-4 months of neoadjuvant chemotherapy followed by radiation in combination with 5-fluorouracil (5FU), gemcitabine, capecitabine, or paclitaxel.

Some studies have evaluated the feasibility and efficacy of FOLFIRINOX or gemcitabine + albumin-bound paclitaxel for neoadjuvant treatment with a small number of patients [15].

\section{Recommendations}

Patients with borderline resectable lesions should be included in clinical trials wherever possible. Acceptable regimens include FOLFIRINOX or gemcitabine + albumin-bound paclitaxel (III, B).

Chemoradiation with gemcitabine or capecitabine-based regimens are another option (III, C).

\section{Locally advanced unresectable disease}

The objective in unresectable locally advanced pancreatic cancer (LAPC) is to increase the survival and quality of life, maintaining local control of the disease. Complications of tumor growth may require decompression maneuvers of the digestive tract or bile duct prior to an active cancer treatment. This must always be accompanied by an appropriate treatment of symptomatic support, initiated at the time of diagnosis [16]. The initial approach is controversial. Radiochemotherapy (RT-QT) improves survival compared to best supportive care or radiotherapy (RT) alone, but with increased toxicity [17]. The LAP07 study showed no benefit in survival when comparing RTQT vs. chemotherapy (CT) in patients with LAPC and controlled after 4 months of induction QT treatment disease, but showed better local control and increased free survival progression [18]. The standard QT treatment in LAPC is gemcitabine, but the significant increase in efficacy with new schedules in metastatic disease (FOLFIRINOX [19] and gemcitabine/nab-Paclitaxel [20]) has lead to their use, also in LAPC, as a reasonable alternative in patients with good performance status (ECOG PS 0-1).

The duration of the initial treatment is not established and depends on tolerability and tumor response.

Using chemoradiotherapy consolidation in patients with response or stabilization after 4-6 months of chemotherapy is an option to consider, vs. maintenance treatment with chemotherapy in patients with good PS. In this context, capecitabine showed a better toxicity profile and efficacy than gemcitabine [21].

\section{Recommendations}

1. The standard treatment for LAPC is chemotherapy with gemcitabine (I, A).

2. By extrapolation of phase III studies in metastatic disease, FOLFIRINOX or gemcitabine/nab-Paclitaxel represents a valid alternative in patients with PS 0-1 and suitable profile of comorbidity $(\mathrm{V}, \mathrm{B})$.

3. After stabilization QT response or induction (4-6 months) consolidation with RT-QT is an alternative to maintaining QT in selected patients (II, B).

\section{Metastatic disease}

In patients with pancreatic carcinoma (PC), it is crucial to evaluate patient-related and disease-related characteristics, such as the intensity of pain (with analogic scales), the 
analgesic requirements, loss of weight (in percentage), the ECOG scale of performance status, the Glasgow Modified Score (that include albumin and protein reactive C), and a geriatric assessment in patients $>70$ years. The geriatric assessment should include comorbidities, cognition, mental health status and support, fatigue, the assessment of polypharmacy, and the presence of geriatric syndromes. Since elderly patients represent a very heterogeneous group, this complex assessment will help to define the frailty or fitness and to guide treatment decisions.

Until 2011, gemcitabine has been considered as the standard of care for patients with metastatic pancreatic cancer [22].

The intense chemotherapeutic regimen of 5-FU, oxaliplatin, and irinotecan (FOLFIRINOX) has been shown superior to GEM monotherapy in PFS and OS [19]. PC patients with mutation in BRAC1, BRAC2 or PALB2 with a prevalence of $10 \%$ would be especially sensitive to this combination, an issue that should be tested prospectively [23]. In the MPACT phase III clinical trial, the combination of gemcitabine and nab-paclitaxel also demonstrated superiority in terms of efficacy for metastatic pancreatic cancer compared with gemcitabine monotherapy [20]. FOLFIRINOX and GEM/nab-paclitaxel are both indicated in fit PC patients with ECOG PS 0, 1 and $<75$ years old. In fit elderly patients ( $>75$ years) or patients with performance status 2, single-agent GEM can be recommended, though in selected patients, gemcitabine and nab-paclitaxel can be considered (Fig. 1). There is no standard chemotherapy for second-line treatment. In patients progressing to gemcitabine or gemcitabine-based combinations, 5-FU/oxaliplatin or 5FU/liposomal irinotecan (NalIri) combination would be considered in selected patients with good PS $[24,25]$. It should be noted that none of these schedules has proven efficacy, in patients progressing to FOLFIRINOX or GEM/nab-paclitaxel. PD1 and PD-L1 inhibitors have not shown activity in PC. PC is a paradigmatic example of a weak immunogenic tumor due to the imbalance between a poor lymphocyte infiltrate (especially CD8) and a high stromal presence that induce a TH2 polarization [26, 27]. Combination of PD-1 or PD-L1 with vaccines or drugs targeting the stroma (either macrophages or fibroblast) deserves to be tested in selected PC patients.

\section{Recommendations}

1. FOLFIRINOX and/or Gem-Abraxane are standard first-line schedules in metastatic disease in patients with ECOG/PS 0-1 (I, A).

2. In selected patients with ECOG 2, gemcitabine and nab-paclitaxel can be considered (II, B).

3. Gemcitabine is an option in elderly patients or patient with PS2 (I, B).

\section{Supportive care}

Patients with pancreatic cancer may experience various distressing symptoms that require ongoing supportive care from diagnosis through treatment (either curative or noncurative). Initial pain management may involve non-opioid drugs, but the mainstay of pain management typically is opiate medication. Celiac plexus block may be used to improve pain relief. It can be performed via percutaneous under ultrasound control, surgical or endoscopic [28]. Patients merit a consultation with an endocrinology or nutritionist because of anorexia and weight loss. Some patients experience exocrine or endocrine pancreatic insufficiency and require pancreatic enzyme replacement and/or insulin therapy [28]. In case of biliary obstruction, the preferred treatment is endoscopic placement of a metal permanent self-expanding stent in the bile duct. Plastic
Fig. $1 *$ Comorbidities, cognition, mental health status and support, fatigue, assessment of polypharmacy, and the presence of geriatric syndromes. **The efficacy of GEM/nabpaclitaxel over gemcitabine in this specific subgroup of patients is currently insufficient

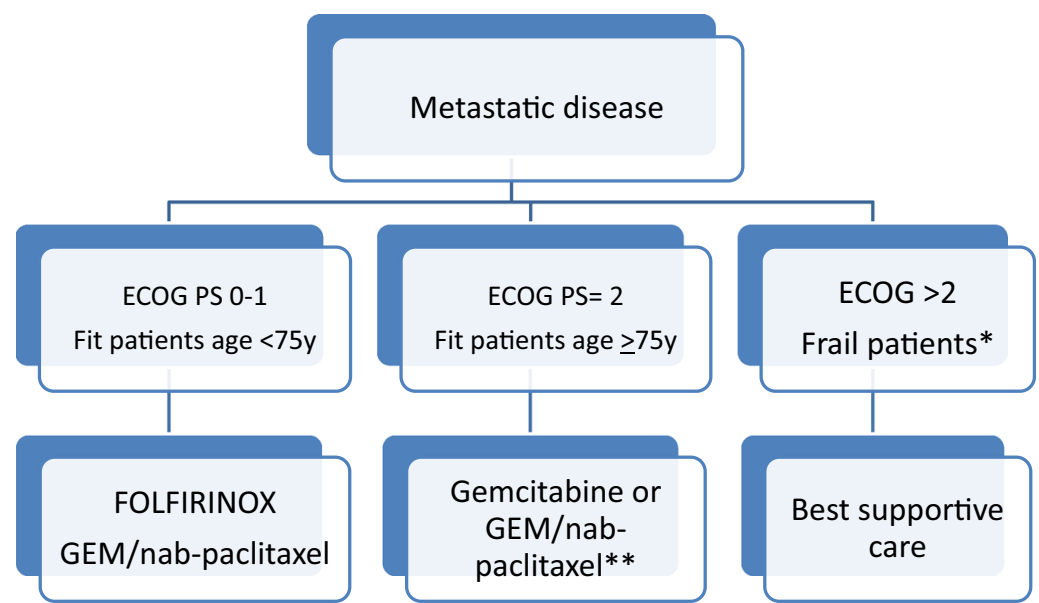


stents can be considered for patients expected to survive $<4$ months [29]. Gastric outlet/duodenal obstruction occur in up to $10 \%$ of patients with pancreatic cancer. Endoscopic duodenal stenting can be successful in the majority of these patients, and median duration of stent patency is 6 months [29]. If venous thromboembolism is presented, in selected high-risk patients (p.e Khorana Index $\geq 3$ ) and no risk of bleeding, prophylaxis with low molecular weight heparin could be considered on a case-by-case basis [30].

\section{Recommendations}

1. Pain management typically is opiate medication (I, A).

2. Biliary and duodenal obstruction can be managed successful with endoscopic stent placement (I, A).

\section{Follow-up}

Five-year survival rate after surgical resection of $\mathrm{PC}$ is $15-25 \%$ and correlates with resection margin status (R0 vs. R1) and lymph node metastases. The majority of recurrences occur within 2 years after resection and can be locoregional and/or to distant sites, including the liver, lung, or peritoneum [31]. Although computed tomography, positron emission tomography and the serum CA19-9 can detect preclinical recurrences, limited available information does not support the idea that early treatment of relapse improves survival [32]. Therefore, considering the poor prognosis of the disease upon diagnosis of a recurrence, there is no evidence that regular follow-up has any impact on the outcome. Follow-up visits should concentrate on symptoms, nutrition, and psycho-social support, resolution of treatment-related toxicity, addressing survivorship issues and monitoring for recurrence [33].

\section{Recommendations}

There is no evidence that regular follow-up after initial therapy with curative intent is useful (IV, D).

\section{Compliance with ethical standards}

Conflict of interest The authors have declared that they have no potential conflicts of interest related to the publication of this manuscript.

Open Access This article is distributed under the terms of the Creative Commons Attribution 4.0 International License (http://crea tivecommons.org/licenses/by/4.0/), which permits unrestricted use, distribution, and reproduction in any medium, provided you give appropriate credit to the original author(s) and the source, provide a link to the Creative Commons license, and indicate if changes were made.

\section{References}

1. De Angelis R, Sant M, Coleman MP, Francisci S, Baili P, Pierannunzio D, EUROCARE-5 Working Group, et al. Cancer survival in Europe 1999-2007 by country and age: results of EUROCARE-5-a population-based study. Lancet Oncol. 2014;15(1):23-34.

2. GLOBOCAN 2012. Estimated cancer incidence, mortality and prevalence Worldwide in 2012. ARCI: OMS. http://globocan.iarc.fr/Default.aspx. último acceso el 16/01/16.

3. Rishi A, Goggins M, Wood LD, Hruban RH. Pathological and molecular evaluation of pancreatic neoplasms. Semin Oncol. 2015;42:28-39.

4. Maisonneuve P, Lowenfels AB. Risk factors for pancreatic cancer: a summary review of meta-analytical studies. Int J Epidemiol. 2015;44:186-98.

5. McIntyre CA, Winter JM. Diagnostic evaluation and staging of pancreatic ductal adenocarcinoma. Semin Oncol. 2015;42(1):19-27.

6. Allen PJ, Kuk D, Castillo CF, Basturk O, Wolfgang CL, Cameron JL, et al. Multi-institutional validation study of the American Joint Commission on Cancer (8th edition) changes for $\mathrm{T}$ and $\mathrm{N}$ staging in patients with pancreatic adenocarcinoma. Ann Surg. (2016) [Epub ahead of print].

7. Al-Hawary MM, Francis IR, Chari ST, Fishman EK, Hough DM, Lu DS, et al. Pancreatic ductal adenocarcinoma radiology reporting template: consensus statement of the Society of Abdominal Radiology and the American Pancreatic Association. Radiology. 2014;270(1):248-60.

8. Pancreatic adenocarcinoma. In: National Comprehensive Cancer Network (NCCN) Practice Guidelines in Oncology, v.1.2016. http://www.ncen.org.

9. Oettle H, Post S, Neuhaus P, Gellert K, Langrehr J, Ridwelski K, et al. Adjuvant chemotherapy with gemcitabine vs observation in patients undergoing curativeintent resection of pancreatic cancer: a randomized controlled trial. JAMA. 2007;297:267-77.

10. Neoptolemus JP, Stocken DD, Bassi C, Ghaneh P, Cunningham D, Goldstein D, et al. Adjuvant chemotherapy with fluorouracil plus folinic acid vs gemcitabine following pancreatic cancer resection: a randomized controlled trial. JAMA. 2010;304:1073-81

11. Neoptolemos J, Palmer D, Ghaneh P, Valle JW, Cunningham D, Wadsley J, et al. ESPAC-4: a multicenter, international, open label randomized controlled phase III trial of adjuvant combination chemotherapy of gemcitabine and capecitabine, versus monotherapy gemcitabine in patients with resected pancreatic ductal adenocarcinoma. J Clin Oncol. 2016;34(abstr LBA 4006).

12. Stocken DD, Büchler, Cervenis C. Meta-analysis of randomized adjuvant therapy trials for pancreatic cancer. Brit J Cancer. 2005;92:1372-81.

13. Katz MH, Pisters PW, Evans DB, Sun CC, Lee JE, Fleming JB, et al. Borderline resectable pancreatic cancer: the importance of this emerging stage of disease. J Am Coll Surg. 2008;206:833-46 (discussion 846-848)

14. Evans DB, Varadhachary GR, Crane CH, Sun CC, Lee JE, Pisters PW, et al Preoperative gemcitabine-based chemoradiation for patients with resectable adenocarcinoma of the pancreatic head. J Clin Oncol. 2008;26:3496-502.

15. Tinchon C, Hubmann E, Pichler A, Keil F, Pichler M, Rabl H, et al. Safety and efficacy of neoadjuvant FOLFIRINOX treatment in a series of patients with borderline resectable pancreatic ductal adenocarcinoma. Acta Oncol. 2013;52:1231-3.

16. Balaban EP, Mangu PB, Khorana AA, Shah MA, Mukherjee S, Crane CH, et al. Locally advanced, unresectable pancreatic cancer: American Society of Clinical Oncology Clinical Practice Guideline. J Clin Oncol. 2016;34(22):2654-68.

17. Shinchi H, Takao S, Noma H, Matsuo Y, Mataki Y, Mori S, et al. Length and quality of survival after external beam radiotherapy with concurrent continuous 5-fluorouracil infusion for locally unresectable pancreatic cancer. Int J Radia Oncol Biol Phys. 2002;53:146-50.

18. Hammel P, Huguet F, Van Laethem JL, Goldstein D, Glimelius B, Artru P, et al. Comparison of chemoradiotherapy (CRT) and chemotherapy (CT) in patients with a locally advanced pancreatic cáncer (LAPC) controlled after 4 months of gemcitabine with or without erlotinib: final results of the international phase III LAP 07 study. J Clin Oncol. 2013;31(suppl):abstr LBA4003.

19. Conroy T, Desseigne F, Ychou M, Bouché O, Guimbaud R, Bécouarn Y, et al. FOLFIRINOX versus gemcitabine for metastatic pancreatic cancer. N Engl J Med. 2011;364:1817-25.

20. Von Hoff DD, Ervin T, Arena FP, Chiorean EG, Infante J, Moore M, et al. Increased survival in pancreatic cancer with nabpaclitaxel plus gemcitabine. N Engl J Med. 2013;369:1691-703.

21. Mukherjee S, Hurt CN, Bridgewater J, Falk S, Cummins S, Wasan H, et al. Gemcitabine-based or capecitabine-based chemoradiotherapy for locally advanced pancreatic cancer (SCALOP): a multicentre, randomised, phase 2 trial. Lancet Oncol. 2013;14:317-26.

22. Burris HA 3rd, Moore MJ, Andersen J, Green MR, Rothenberg ML, Modiano $\mathrm{MR}$, et al. Improvements in survival and clinical benefit with gemcitabine as first-line therapy for patients with advanced pancreas cancer: a randomized trial. J Clin Oncol. 1997;15:2403-13.

23. Waddell N, Pajic M, Patch AM, Chang DK, Kassahn KS, Bailey P, et al. Whole genomes redefine the mutational landscape of pancreatic cancer. Nature. 2015;518:495-501. 
24. Oettle H, Riess H, Stieler JM, Heil G, Schwaner I, Seraphin J, et al. Second-line oxaliplatin, folinic acid, and fluorouracil versus folinic acid and fluorouracil alone for gemcitabine-refractory pancreatic cancer: outcomes from the CONKO-003 trial. J Clin Oncol. 2014;32:2423-9.

25. Wang-Gillam A, Li CP, Bodoky G, Dean A, Shan YS, Jameson G, NAPOLI-1 Study Group, et al. Nanoliposomal irinotecan with fluorouracil and folinic acid in metastatic pancreatic cancer after previous gemcitabine-based therapy (NAPOLI-1): a global, randomised, open-label, phase 3 trial. Lancet. 2016;387:545-57.

26. Feig C, Jones JO, Kraman M, Wells RJ, Deonarine A, Chan DS, et al. Targeting CXCL12 from FAP-expressing carcinoma-associated fibroblasts synergizes with anti-PD-L1 immunotherapy in pancreatic cancer. Proc Natl Acad Sci USA. 2013;110:20212-7.

27. Gunderson AJ, Kaneda MM, Tsujikawa T, Nguyen AV, Affara NI, Ruffell B, et al. Bruton tyrosine kinase-dependent immune cell cross-talk drives pancreas cancer. Cancer Discov. 2016;6:270-8.
28. Sohal DP, Mangu PB, Khorana AA, Shah MA, Philip PA, O'Reilly EM Metastatic pancreatic cancer: American Society of Clinical Oncology Clinical Practice Guideline. J Clin Oncol. 2016;34(23):2784-96.

29. Stark A, Hines OJ. Endoscopic and operative palliation strategies for pancreatic ductal adenocarcinoma. Semin Oncol. 2015;42(1):163-76.

30. Lyman GH, Bohlke K, Falanga A. Venous thromboembolism prophylaxis and treatment in patients with cancer: American Society of Clinical Oncology Clinical Practice Guideline Update. J Oncol Pract. 2015;11:e442-4.

31. Castellanos JA, Merchant NB. Intensity of follow-up after pancreatic cancer resection. Ann Surg Oncol. 2014;21:747-51.

32. Furman MJ, Lambert LA, Sullivan ME, Whalen GF. Rational follow-up after curative cancer resection. J Clin Oncol. 2013;31:1130-3.

33. Ducreux M, Cuhna AS, Caramella C, Hollebecque A, Burtin P, Goéré D, et al. Cancer of the pancreas: ESMO clinical practice guidelines for diagnosis, treatment and follow-up. Ann Oncol. 2015;26(Supplement 5):v56-68. 